\title{
Recent endemic coronavirus infection is associated with less-severe COVID-19
}

\author{
Manish Sagar, ${ }^{1}$ Katherine Reifler, ${ }^{1}$ Michael Rossi, ${ }^{1}$ Nancy S. Miller, ${ }^{2}$ Pranay Sinha, ${ }^{1}$ Laura F. White, ${ }^{3}$ and Joseph P. Mizgerd ${ }^{1}$ \\ 'Department of Medicine and ${ }^{2}$ Department of Pathology and Laboratory Medicine, Boston University School of Medicine, Boston, Massachusetts, USA. ${ }^{3}$ Department of Biostatistics, Boston University School \\ of Public Health, Boston, Massachusetts, USA.
}

\begin{abstract}
Four different endemic coronaviruses (eCoVs) are etiologic agents for the seasonal common cold, and these eCoVs share extensive sequence homology with human SARS coronavirus 2 (SARS-CoV-2). Here, we show that individuals with, as compared with those without, a recent documented infection with eCoV were tested at greater frequency for respiratory infections but had a similar rate of SARS-CoV-2 acquisition. Importantly, the patients with a previously detected eCoV had less-severe coronavirus disease 2019 (COVID-19) illness. Our observations suggest that preexisting immune responses against endemic human coronaviruses can mitigate disease manifestations from SARS-CoV-2 infection.
\end{abstract}

\section{Introduction}

While SARS coronavirus 2 (SARS-CoV-2) emerged recently, other coronaviruses are endemic in the human population. Four different human coronaviruses (HCoV-OC43, HCoV-HKU1, HCoVNL63, and HCoV-229E) are among the most common etiologic agents for the seasonal common cold and also cause pneumonia $(1,2)$. SARS-CoV-2-induced disease, termed coronavirus disease 2019 (COVID-19), can vary from asymptomatic to acute respiratory distress syndrome requiring mechanical ventilation or leading to death $(3,4)$. The endemic coronaviruses (eCoVs) share extensive sequence homology with SARS-CoV-2, and immune responses to the eCoVs can cross-react with SARS-CoV-2 antigens (5-8). Whether prior infection with eCoV elicits immunologic memory that influences SARS-CoV-2 acquisition and COVID-19 outcomes remains uncertain.

\section{Results and Discussion}

We examined SARS-CoV-2 infections and COVID-19 outcomes among patients who had previously been assessed with a comprehensive respiratory panel PCR (CRP-PCR) test (FilmArray Respiratory Panel [RP2], BioFire Diagnostics). CRP-PCR detects nucleic acids for the 4 eCoVs along with 16 other pathogens, and thus, a positive test indicates ongoing rather than prior infections. We retrospectively collected data from patients with an available CRP-PCR result from May 18, 2015, to March 11, 2020, in the electronic medical record (EMR). March 11, 2020, was chosen as the end date because the first available SARS-CoV-2 test in the Boston Medical Center (BMC) EMR was on March 12, 2020. We also obtained all SARS-CoV-2 reverse transcription PCR (RT-PCR) results between March 12, 2020, and June 12, 2020, that were available in the EMR. Analysis was restricted to patients not

Conflict of interest: The authors have declared that no conflict of interest exists. Copyright: (5) 2021, American Society for Clinical Investigation.

Submitted: August 18, 2020; Accepted: September 29, 2020; Published: January 4, 2021.

Reference information: J Clin Invest. 2021;131(1):e143380.

https://doi.org/10.1172/JCl143380. recorded deceased prior to March 11, 2020, older than 18 years, and with the first SARS-CoV-2 result documented at least 7 days after the CRP-PCR test.

A total of 15,928 patients had at least 1 CRP-PCR test. An eCoV was previously detected in 875 of these patients (termed $\mathrm{eCoV}^{+}$), and the remaining 15,053 individuals (classified as $\mathrm{eCoV}^{-}$) had never had a documented eCoV infection. For most, but not all, demographic characteristics, there was no significant difference between the $\mathrm{eCoV}^{+}$and $\mathrm{eCoV}^{-}$groups (Table 1), although there were some variations in race and HIV infection status. The proportion of patients with no, 1 , or 2 or more comorbidities was not significantly different between the $\mathrm{eCoV}^{+}$and $\mathrm{eCoV}^{-}$groups. The CRP-PCR test was more frequently ordered while patients were at a hospital (inpatient, observation unit, or emergency department) in the $\mathrm{eCoV}^{-}$as compared with the $\mathrm{eCoV}^{+}$group. These observations imply that the patients in the 2 groups had a similar level of preexisting morbidity, but the $\mathrm{eCoV}^{-}$as compared with the $\mathrm{eCoV}^{+}$ patients may have had more severe clinical presentation at the time of CRP-PCR testing.

A total of 1812 (11.4\%) of the patients under investigation had an available SARS-CoV-2 result (Table 2). A significantly higher proportion of $\mathrm{eCoV}^{+}(15.2 \%)$ individuals were tested for SARSCoV-2 as compared with $\mathrm{eCoV}^{-}(11.2 \%)$ patients (OR 1.4, 95\% CI 1.2-1.7). The odds of SARS-CoV-2 testing (OR 1.4, 95\% CI 1.2-1.7) remained significantly higher in the $\mathrm{eCoV}^{+}$as compared with the $\mathrm{eCoV}^{-}$patients after adjusting for race/ethnicity, chronic obstructive pulmonary disease, HIV, number of comorbidities, and level of clinical care. The last documented CRP-PCR result prior to the SARS-CoV-2 RT-PCR test occurred significantly more recently in the $\mathrm{eCoV}^{+}$(median 121 days, IQR 69-440 days) as compared with the $\mathrm{eCoV}^{-}$patients (median 359 days, IQR 117-799 days; $P<$ 0.0001) (Figure 1A). The $\mathrm{eCoV}^{+}$(median 2, IQR 1-3) as compared with the $\mathrm{eCoV}^{-}$(median 1, IQR 1-2; $P=0.002$ ) patients also had significantly more frequent CRP-PCR testing (Figure 1B). The more recent and frequent CRP-PCR testing in the $\mathrm{eCoV}^{+}$individuals suggests a greater likelihood of having a clinical presentation prompting respiratory evaluation. The greater likelihood of illness 
Table 1. Demographics of patients with and without a documented eCoV

\begin{tabular}{|c|c|c|c|}
\hline & eCoV- $(n=15,053)$ & $\mathrm{eCoV}+(n=875)$ & $P$ value \\
\hline Age, median (IQR) & $55(38-68)$ & $55(37-68)$ & $0.34^{\mathrm{A}}$ \\
\hline Male/female & $6938(46.1) / 8115(53.9)$ & $421(48.1) / 454$ (51.9) & 0.25 \\
\hline Race/ethnicity & & & $0.05^{\mathrm{B}}$ \\
\hline Black & $6757(44.9)$ & $365(41.7)$ & \\
\hline White & $4311(28.6)$ & $248(28.3)$ & \\
\hline Hispanic/Latino & $3,282(21.8)$ & $219(25.0)$ & \\
\hline BMI, median (IQR) & $27.9(23.8-32.8)$ & $27.8(23.9-32.8)$ & $0.59^{A}$ \\
\hline DM & $4481(29.8)$ & $270(30.9)$ & 0.49 \\
\hline Hypertension & $7525(50.0)$ & $443(50.6)$ & 0.73 \\
\hline Coronary artery disease & $1515(10.1)$ & $87(9.9)$ & 0.95 \\
\hline Congestive heart failure & $1311(8.7)$ & $77(8.8)$ & 0.90 \\
\hline Chronic obstructive pulmonary disease & $2342(15.6)$ & $151(17.3)$ & 0.18 \\
\hline Asthma & $3583(23.8)$ & $216(24.7)$ & 0.54 \\
\hline Renal disease & $1681(11.2)$ & $103(11.8)$ & 0.58 \\
\hline Human immunodeficiency virus & $659(4.4)$ & $53(6.1)$ & 0.02 \\
\hline Cancer & $1459(9.7)$ & $93(10.6)$ & 0.38 \\
\hline End-stage renal disease & $464(3.1)$ & $33(3.8)$ & 0.27 \\
\hline Number of comorbidities & & & $0.18^{B}$ \\
\hline 0 & $4298(28.6)$ & $244(27.9)$ & \\
\hline 1 & 3892 (25.9) & $206(23.5)$ & \\
\hline$\geq 2$ & $6863(45.6)$ & $425(48.6)$ & \\
\hline Level of clinical care ${ }^{c}$ & & & $<0.0001^{1}$ \\
\hline Inpatient & $7,047(46.8)$ & $331(37.8)$ & \\
\hline Observation unit & $2681(17.8)$ & $134(15.3)$ & \\
\hline Emergency department & $4118(27.4)$ & $308(35.2)$ & \\
\hline Outpatient & $1174(7.8)$ & $99(11.3)$ & \\
\hline Missing data & $33(0.2)$ & $3(0.3)$ & \\
\hline
\end{tabular}

Race/ethnicity is based on patient-supplied information (some patients did not provide such information), and diagnoses represent the most current problem listed in the medical record. Data are expressed as number (\%), and $P$ value was calculated using Fisher's exact test unless otherwise indicated. ${ }^{A}$ Mann-Whitney $U$ test. ${ }^{B} \chi^{2}$ test. ' Level of clinical care at the time of the CRP-PCR test. tested, and of hospitalization among those infected, did not differ between the $\mathrm{eCoV}^{+}$and $\mathrm{eCoV}^{-}$ groups (Table 2). Some risk factors associated with more severe COVID-19, such as older age, male sex, higher BMI, and preexisting diabetes mellitus (DM) $(9,10)$, were significantly different between the $\mathrm{eCoV}^{+}$and $\mathrm{eCoV}^{-}$patients who were eventually hospitalized after SARS-CoV-2 infection (Supplemental Table 1; supplemental material available online with this article; https://doi.org/10.1172/ JCI143380DS1). The numbers of prior diagnoses, however, were not different among the hospitalized $\mathrm{eCoV}^{+}$and $\mathrm{eCoV}^{-}$groups, suggesting they had a similar level of preexisting morbidity.

The $\mathrm{eCoV}^{+}$as compared with the $\mathrm{eCoV}^{-}$hospitalized patients had a significantly lower odds for intensive care unit (ICU) admission (OR $0.1,95 \%$ CI $0.0-0.7)$ and a trend toward lower odds of mechanical ventilation (OR 0.0, 95\% CI 0.0-1.0). The odds of ICU care (OR 0.1, 95\% CI 0.1-0.9) remained significantly lower in the $\mathrm{eCoV}^{+}$as compared with the $\mathrm{eCoV}^{-}$patients after adjustment for age, sex, BMI, and DM status. The percentage of hospitalized patients who eventually died over follow-up was lower in the $\mathrm{eCoV}^{+}$ $(4.8 \%)$ as compared with the $\mathrm{eCoV}^{-}(17.7 \%)$ group. Survival probability was significantly higher in the $\mathrm{eCoV}^{+}$than the $\mathrm{eCoV}^{-}$hospitalized COVID-19positive patients (HR 0.3, 95\% CI 0.1-0.7; Figure 2). After adjustment for age, sex, BMI, and DM, the HR remained 0.3, although the CI became much wider and encompassed unity (0.0 to 2.0). Cumulatively, these observations suggest that recent documented $\mathrm{eCoV}$ infection is associated with less-severe COVID-19.

Lower virus levels in the respiratory tract assoprompting CRP-PCR evaluation may also account for the higher level of SARS-CoV-2 RT-PCR testing among the $\mathrm{eCoV}^{+}$as compared with the $\mathrm{eCoV}^{-}$group.

Among the patients evaluated for SARS-CoV-2, 470 (25.9\%) had at least 1 positive SARS-CoV-2 RT-PCR at some point (Table 2). A total of 252 (53.6\%) of the SARS-CoV-2-infected patients had a COVID-19-related hospitalization during the study period. The frequency of documented SARS-CoV-2 infection among those ciate with less-severe COVID-19 (11). The EMR provides scant information from which a patient's burden of infection may be inferred, but $\mathrm{Ct}$ values from SARS-CoV-2 tests may be used for extrapolation. Both a commercial Abbott assay and an in-house assay (12) were used to determine the presence of SARS-CoV-2 in our hospital during this study period due to testing and material limitations. Patient $\mathrm{Ct}$ values did not differ significantly according to which test was used ( $P=0.13$; Supplemental Figure 1A). In the patient's initial

\section{Table 2. SARS-CoV-2 infection and COVID-19 outcomes in patients with and without a documented eCoV}

\begin{tabular}{|c|c|c|c|c|}
\hline & eCoV- $(n=15,053)$ & $\mathrm{eCoV}^{+}(n=875)$ & OR (95\% Cl) eCoV $/ \mathrm{eCoV}^{-}$ & Adjusted OR ( $95 \% \mathrm{Cl})$ \\
\hline SARS-CoV-2 tested, $n$ ( $\%$ of total) & $1679(11.2)$ & $133(15.2)$ & $1.4(1.2-1.7)$ & $1.4(1.2-1.7)^{A}$ \\
\hline SARS-CoV- $2^{+}, n$ ( $\%$ of tested) & $437(26.0)$ & $33(24.8)$ & $0.9(0.6-1.4)$ & \\
\hline Hospitalized, $n$ ( $\%$ of SARS-CoV- $\left.2^{+}\right)$ & $231(52.9)$ & $21(63.6)$ & $1.6(0.8-3.2)$ & \\
\hline Mechanical ventilation, $n$ ( $\%$ of hospitalized) & $38(16.4)$ & $0(0)$ & $0.0(0.0-1.0)$ & \\
\hline
\end{tabular}

OR was calculated using Fisher's exact test. ${ }^{A} \mathrm{OR}$ after adjusting for race/ethnicity, chronic obstructive pulmonary disease, HIV, number of comorbidities, and level of clinical care using multivariate logistic regression. ${ }^{B} \mathrm{OR}$ after adjusting for age, sex, BMI, and DM using penalized logistic regression. 


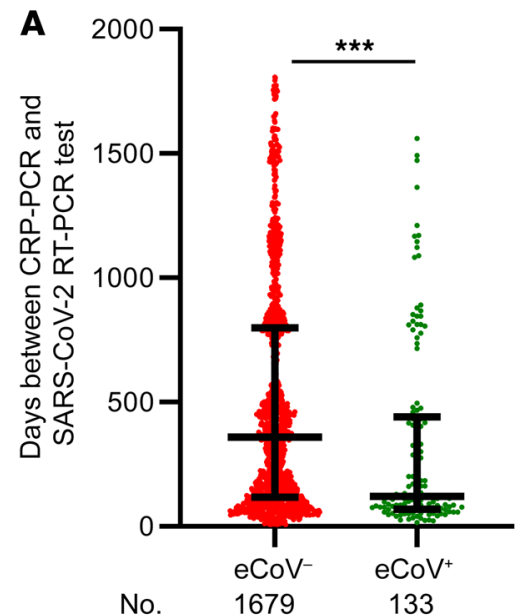

Figure 1. Testing among patients with and without a documented eCoV. Days between the last available CRP-PCR and first SARS-CoV-2 RT-PCR test (A) and number of independent CRP-PCR tests from May 18, 2015, to March 11, 2020 (B) among $\mathrm{eCoV}^{-}$and $\mathrm{eCoV}^{+}$patients. The numbers of patients (No.) contributing to the data are indicated. The black lines in the dot plots represent median and IQR. ${ }^{* *} P<0.01,{ }^{* * *} P<0.001$, Mann-Whitney $U$ test. or only positive SARS-CoV-2 RT-PCR, virus in the nasophayrnx was more abundant in $\mathrm{eCoV}^{+}$as compared with $\mathrm{eCoV}^{-}$patients, as indicated by lower Ct values (Supplemental Figure 1B). A multivariable linear regression model demonstrated that the $\mathrm{eCoV}^{+}$as compared with the $\mathrm{eCoV}^{-}$patients had a Ct value of around 4 units lower ( $\beta=4.0,95 \% \mathrm{CI}-0.6-8.7, P=0.09)$, but this difference was not statistically significant. The in-house assay also trended toward yielding Ct values around 2 units lower than those from the commercial Abbott assay $(\beta=2.0,95 \% \mathrm{CI}-0.3-4.4, P=0.09)$. In both analyses, the differences did not reach statistical significance, and the number of data points was extremely limited, especially for the $\mathrm{eCoV}^{+}$ patients. These data do not support the hypothesis that the better outcomes in the $\mathrm{eCoV}^{+}$patients were due to lower viral burden. This conclusion, however, is limited by small sample size, single as opposed to longitudinal sampling, and assessment of only the upper and not the lower airway. Combined with the SARS-CoV-2 acquisition frequency data (Table 2), these observations potentially imply that the $\mathrm{eCoV}^{+}$patients did not possess immunological memory that constrained initial virus replication.

Less-severe outcomes from similar viral loads may be due to decreased cytokine storm or inflammatory injury. Higher levels of plasma inflammatory markers, such as C-reactive protein (CRP) and lactate dehydrogenase (LDH), correlate with more severe disease (13), supporting the concept that inflammatory responses contribute to pathophysiology. The $\mathrm{eCoV}^{+}$as compared with the $\mathrm{eCoV}^{-}$patients trended toward lower levels of CRP $\left(\mathrm{eCoV}^{+}\right.$ median $24.0 \mathrm{ng} / \mathrm{L}$, IQR 7.2-69.3 ng/L versus $\mathrm{eCoV}^{-}$median 55.1 ng/L, IQR 16.6-109.0 ng/L; $P=0.06)$ and $\mathrm{LDH}\left(\mathrm{eCoV}^{+}\right.$median 284.0 U/L, IQR 191-344.5 U/L versus $\mathrm{eCoV}^{-}$median $306.0 \mathrm{U} / \mathrm{L}$, IQR 241-385.5 U/L; $P=0.09$ ) upon their initial presentation for COVID-19-related hospitalization (Supplemental Figure 2, A and B). These observations possibly suggest that patients with a previously documented $\mathrm{eCoV}$ infection may have more subdued inflammatory responses soon after SARS-CoV-2 infection $(14,15)$.

As a whole, patients with CRP-PCR tests prior to SARS-CoV-2 acquisition are hospitalized at a higher frequency as compared with the general population at our and other medical centers (16, 17). This population may represent a group with a higher propensity to acquire a respiratory infection and require hospitalization. We found that, within this population, individuals with a recent prior documented eCoV infection were more likely to have a clinical presentation triggering SARS-CoV-2 testing, but their likelihood of being infected was similar. The level of hospitalization after infection also did not differ between the 2 groups. We interpret these data to suggest that those with recent eCoV infection may not have neutralizing immunity that prevents acquisition. Indeed, a previous study suggests that neutralizing responses against eCoVs are relatively short lived, and previously infected patients are susceptible to reinfection, albeit with less-severe disease (18). Importantly, we observed that the $\mathrm{eCoV}^{+}$as compared
Figure 2. Mortality among patients with and without a documented eCoV. Unadjusted Kaplan-Meier survival curve for $\mathrm{eCoV}^{-}$and eCoV+ SARS-CoV-2infected hospitalized patients. The $y$ axis shows the probability of survival, and the $x$ axis shows days after first SARS-CoV-2-positive RT-PCR result. The tick marks denote right censoring after July 14, 2020. The number of patients at risk at different time points is shown. Unadjusted $(0.3,95 \% \mathrm{Cl}$ $0.1-0.7)$ and adjusted $(0.3,95 \% \mathrm{Cl} 0.0-2.0)$ survival HRs were calculated using the log rank test and Cox's proportional hazard model, respectively.

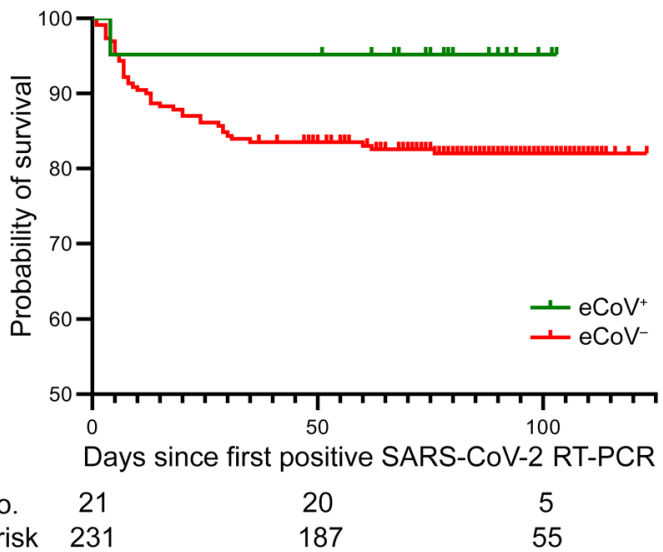


with the $\mathrm{eCoV}^{-}$group had lower rates of ICU admission and death after COVID-19 diagnosis. Even without neutralizing immunity, patients with prior eCoV infections may have lung-localized primed immune responses that prevent severe disease from a heterologous virus (19). Heterotypic lung-localized resident memory $\mathrm{T}$ and $\mathrm{B}$ cells prevent severe infections from respiratory pathogens (20). Future studies should determine whether lung-localized heterotypic immunity is elicited by prior eCoV infection and is capable of ameliorating COVID-19 manifestations. The durability and extent of the potential immune protection and distinct effects of different eCoVs will also need to be investigated.

This study had limitations. It was associative, and thus cannot determine causality. It involved small numbers from one hospital, so findings may not generalize. The observed morbidity and mortality may be linked to but not directly caused by SARS-CoV-2 infection. The absence of an $\mathrm{eCoV}^{+}$result does not preclude coronavirus infections throughout the study period, so some individuals may have been classified inappropriately. The relatively low observed morbidity and mortality in the $\mathrm{eCoV}^{+}$group, however, suggest that removing individuals with undocumented $\mathrm{eCoV}$ infection from the $\mathrm{eCoV}^{-}$group would further increase the effect size away from the null. Several different RT-PCR assays were used for SARS-CoV-2 testing at our institution during the study period; inherent differences in their targets and Ct values are acknowledged. With these limitations, results suggest that prior eCoV infection was associated with less-severe COVID-19. Larger studies and causal investigations are needed to identify the mechanisms and persistence of this inferred heterotypic immune protection.

\section{Methods}

Patient data. All data were obtained from patients' EMR. All test results were based on clinical care, and no tests were done for research purposes. All tests and clinical care were at the discretion of the treating physicians. There were no limitations or perquisites for CRP-PCR testing at BMC. A patient with a documented $\mathrm{eCoV}$ on CRP-PCR was classified as $\mathrm{eCoV}^{+}$regardless of whether they had other CRP-PCR results. All other patients were classified as $\mathrm{eCoV}^{-}$. The test date of interest was the day with documented $\mathrm{eCoV}$ for the $\mathrm{eCoV}^{+}$group and the most recent $\mathrm{CRP}^{-} \mathrm{PCR}$ for the $\mathrm{eCoV}^{-}$group. For each patient, we also recorded the day of the most recent CRP-PCR result. The first positive SARS-CoV-2 RT-PCR result was designated as the SARS$\mathrm{CoV}-2$ test day regardless of whether the patient had other results. For the patients with negative SARS-CoV-2 RT-PCR results, the first negative test was designated as the SARS-CoV-2 test day. An individual patient was only counted once regardless of the number of CRP-PCR or SARS-CoV-2 test results. All data from patients younger than 18 years were excluded from the analysis.

Quantitative SARS-CoV-2 RT-PCR testing. RT-PCR Ct values were obtained from a commercial Abbott assay (Abbott RealTime SARSCoV-2 for m2000 RT-PCR) and an in-house assay (12). The Abbott assay gene target is proprietary. The in-house assay targeted the SARS-
CoV-2 nucleocapsid gene. The Ct values obtained from the different assays were examined using multivariable linear regression. In this model, $\mathrm{eCoV}^{+}$versus $\mathrm{eCoV}^{-}$(group) and the Abbott versus the in-house assay (platform) were categorical independent variables, and Ct was the dependent variable. An interaction term between the group and platform variable did not improve the model, and thus it was omitted from the final analysis.

Statistics. Analyses were conducted using GraphPad Prism (version 8.4.3) and SPSS Statistics (version 26.0). Descriptive statistics were used to summarize the data and report medians and IQRs as appropriate. Outcomes of interest were proportion SARS-CoV-2 tested, SARS-CoV-2 positivity, hospitalization, ICU admission, mechanical ventilation, and death. Patient characteristics were assessed using Fisher's exact, Mann-Whitney $U$, and $\chi^{2}$ tests. Unadjusted and adjusted ORs were estimated using Fisher's exact tests and multivariate or penalized likelihood logistic regression, respectively. Mortality rate differences were compared using log rank HR and multivariate Cox's proportional hazard analysis. All patients were right censored after July 14, 2020. Multivariate comparisons incorporated characteristics deemed important for COVID-19 and those demonstrating a $P$ value less than 0.2 in univariate analyses. Tests were 2 sided, with a $P$ value less than 0.05 considered statistically significant.

Study approval. This retrospective study did not require patient consent and was approved by the Boston University Institutional Review Board (H40391).

\section{Author contributions}

JPM conceived the study. MS and JPM designed the study. KR, $\mathrm{MR}$, and MS collected data. NSM provided the quantitative PCR data. MS, PS, and LFW conducted the statistical analyses. MS, PS, LFW, and JPM analyzed and interpreted the data. MS and JPM wrote the manuscript.

\section{Acknowledgments}

We thank Aditya Mithal for assistance in obtaining the SARSCoV-2 RT-PCR Ct values. This work was supported by grants from the NIH (R35 HL-135756 and R01 AI-115053 to JPM, K24 AI-145661 to MS, 5T32 AI-052074-13 to PS, and R01 GM-122876 to LFW). The funding organization had no role in the design or conduct of the study; collection, management, analysis, or interpretation of the data; preparation, review, or approval of the manuscript; or decision to submit the manuscript for publication. The work was facilitated by the Providence/Boston Center for AIDS Research (P30AI042853).

Address correspondence to: Manish Sagar, Boston University School of Medicine, 650 Albany Street, Room 647, Boston, Massachusetts 02118, USA. Phone: 617.414.5239; Email: msagar@bu.edu. Or to: Joseph P. Mizgerd, Boston University School of Medicine, Pulmonary Center, 72 East Concord Street, Boston, Massachusetts 02118, USA. Phone: 617.358.1186; Email: jmizgerd@bu.edu.
1. Sariol A, Perlman S. Lessons for COVID-19 immunity from other coronavirus infections. Immunity. 2020;53(2):248-263.

2. Jain S, et al. Community-acquired pneumonia requiring hospitalization among U.S. adults.
N Engl J Med. 2015;373(5):415-427.

3. Wu JT, et al. Estimating clinical severity of COVID-19 from the transmission dynamics in

Wuhan, China. Nat Med. 2020;26(4):506-510.

4. Oran DP, Topol EJ. Prevalence of asymptomatic
SARS-CoV-2 infection : a narrative review. Ann Intern Med. 2020;173(5):362-367.

5. Le Bert N, et al. SARS-CoV-2-specific T cell immunity in cases of COVID-19 and SARS, and uninfected controls. Nature. 
2020;584(7821):457-462.

6. Grifoni A, et al. Targets of $\mathrm{T}$ cell responses to SARS-CoV-2 coronavirus in humans with COVID-19 disease and unexposed individuals. Cell. 2020;181(7):1489-1501.e15.

7. Wec AZ, et al. Broad neutralization of SARSrelated viruses by human monoclonal antibodies. Science. 2020;369(6504):731-736.

8. Braun J, et al. SARS-CoV-2-reactive T cells in healthy donors and patients with COVID-19. Nature. 2020;587(7833):270-274.

9. Wu C, et al. Risk factors associated with acute respiratory distress syndrome and death in patients with coronavirus disease 2019 pneumonia in Wuhan, China. JAMA Intern Med. 2020;180(7):934-943.

10. Richardson $S$, et al. Presenting characteristics, comorbidities, and outcomes among 5700 patients hospitalized with COVID-19 in the New York City Area. JAMA. 2020;323(20):2052-2059.
11. Liu Y, et al. Viral dynamics in mild and severe cases of COVID-19. Lancet Infect Dis. 2020;20(6):656-657.

12. Vanuytsel K, et al. Rapid implementation of a SARS-CoV-2 diagnostic quantitative real-time PCR test with emergency use authorization at a large academic safety net hospital [Published online May 19, 2020]. Med (N Y). https://doi. org/10.1016/j.medj.2020.05.001.

13. Chen G, et al. Clinical and immunological features of severe and moderate coronavirus disease 2019. J Clin Invest. 2020;130(5):2620-2629.

14. Blanco-Melo D, et al. Imbalanced host response to SARS-CoV-2 drives development of COVID19. Cell. 2020;181(5):1036-1045.e9.

15. Hadjadj J, et al. Impaired type I interferon activity and inflammatory responses in severe COVID-19 patients. Science. 2020;369(6504):718-724.

16. Hsu HE, et al. Race/ethnicity, underlying medical conditions, homelessness, and hospitalization status of adult patients with COVID-19 at an Urban Safety-Net Medical Center - Boston, Massachusetts, 2020. MMWR Morb Mortal Wkly Rep. 2020;69(27):864-869.

17. Garg S, et al. Hospitalization rates and characteristics of patients hospitalized with laboratoryconfirmed coronavirus disease 2019 - COVIDNET, 14 States, March 1-30, 2020. MMWR Morb Mortal Wkly Rep. 2020;69(15):458-464.

18. Callow KA, Parry HF, Sergeant M, Tyrrell DA. The time course of the immune response to experimental coronavirus infection of man. Epidemiol Infect. 1990;105(2):435-446.

19. Hua X, et al. Nasal priming by a murine coronavirus provides protective immunity against lethal heterologous virus pneumonia. JCI Insight. 2018;3(11):e99025.

20. Quinton LJ, Walkey AJ, Mizgerd JP. Integrative physiology of pneumonia. Physiol Rev. 2018;98(3):1417-1464. 